\title{
Erratum to: Approximating adaptive distance measures using scalable feature signatures
}

\section{Jakub Lokoč}

Published online: 28 April 2015

(C) Springer Science+Business Media New York 2015

\section{Erratum to: Multimed Tools Appl \\ DOI 10.1007/s11042-014-2251-4}

The original article contained an incorrect equation in "3 Scalable feature signatures," under the section Total Orderings. The equation corresponding to the distance function inspired by the Ward's method missed a square in the expression and also repeated the delta symbol in the lower index. The correct equation is shown below:

$$
\delta_{W L_{2}}\left(t_{i}, t_{j}\right)=L_{2}^{2}\left(r_{i}, r_{j}\right)\left(w_{i} w_{j}\right) /\left(w_{i}+w_{j}\right)
$$

The online version of the original article can be found at http://dx.doi.org/10.1007/s11042-014-2251-4.

J. Lokoč $(\bowtie)$

SIRET research group, Department of Software Engineering, Faculty of Mathematics and Physics,

Charles University in Prague, Prague, Czech Republic

e-mail: lokoc@ksi.mff.cuni.cz 